\title{
Citric Acid Catalyzed Deprotection of Carbonyl Compounds from Phenylhydrazones, Semicarbazones and Oximes Under Microwave Irradiations
}

\author{
NAGLAA M. ABD EL RAHMAN ${ }^{1,2}$ and AAYESHA NASREEN *1 \\ 'Department of Chemistry, College of Sciences, Jazan University, Saudi Arabia. \\ ${ }^{2}$ Green Chemistry Department, National Research Center, 33 ELBohouth St. \\ (former El Tahrir St.), Dokki, Giza 12622, Egypt. \\ ${ }^{*}$ Corresponding author E-mail: aayesha_iict@yahoo.co.in \\ http://dx.doi.org/10.13005/ojc/330451
}

(Received: May 07, 2017; Accepted: July 02, 2017)

\begin{abstract}
Deprotection of phenylhydrazones, semicarbazones and oximes to their corresponding carbonyl compounds have been carried out in good to excellent yields (83-96\%) by using citric acid as organic catalyst in water as a medium of reaction under microwave irradiation.
\end{abstract}

Keywords: Imine derivatives, Microwave chemistry, Carbonyl compounds, Citric acid

\section{INTRODUCTION}

The protection of the carbon-oxygen double bonds as carbon-nitrogen double bonds has become a useful methodology in the synthesis of complex organic molecules ${ }^{1}$. Semicarbazones, phenylhydrazones and oximes are highly stable and readily prepared compounds, which are used extensively for the protection of carbonyl groups ${ }^{2}$ and are also used for the purification and characterization of carbonyl compound ${ }^{3}$. The cleavage of imine derivatives to afford carbonyl compounds continues to be a significant aspect of organic chemical transformations.
Most of the known methods deal for the regeneration of carbonyl compounds from oximes only, few publications were reported for phenylhydrazones and semicarbazones. Therefore search for faster, new protocols for the regeneration of carbonyl compounds from phenylhydr-azones, semicarbazones and oximes has assumed added importance.

On the other hand, some of these reported methods were carried out under conventional methods or microwave irradiations in the presence of oxidative or reductive catalysts. For example $\mathrm{Al}_{2} \mathrm{O}_{3}{ }^{4}$, polymer beads of $\mathrm{N}, \mathrm{N}$-dich-loropoly 
(styrene-co-divinylbenzene) sulphonamide resin ${ }^{5}$, Sodium periodate- $\mathrm{SiO}_{2}{ }^{6}$, hypervalent iodine, bis[dipyrid-inesilver (I) dichromate ${ }^{8}$, Manganese triacetate $^{9}$, urea nitrate ${ }^{10}$, and low valent titanium ${ }^{11}$, $\mathrm{MnO}_{2}{ }^{12}$, Clay ferric nitrate ${ }^{13}, 2,6$-dicarboxpyri diniumchlorochromate ${ }^{14}$, Copper (II) Chloride dehydrate $^{15}$, Wetsilica-supported Perm-anganate ${ }^{16}$, CAN-SiO ${ }_{2}{ }^{17}$, Formic acid ${ }^{18}, \mathrm{Ag}($ py $){ }_{4} \mathrm{~S}_{2} \mathrm{O}_{8}{ }^{19}$, $\mathrm{SiBr}_{4}{ }_{4}^{20}$, BNBTS $^{21}$, Thalium(III) ${ }^{22}$, Lead(IV) ${ }^{23}$, Chromium $(\mathrm{VI})^{24}$, Zeolite H-NaX Supported Copper nitrate ${ }^{25}$. It is worthy to mention that most of these reagents are toxic or expensive.

Organic chemists are interested in the developing of ecofriendly procedures by using microwave irradiation under solvent free conditions in water as a green solvent. The advantages of these methods are shorter reaction time, excellent yields, besides, formation of cleaner products and minimization of pollutants ${ }^{26-28}$.

Make use of simply available and biodegradable catalyst for organic conversion is achieving enormous significance in the last few years as a result of both the uniqueness of the concept and, more importantly, the fact that the efficiency and selectivity of these reactions meet the standards of established organic reactions. In this regard, Citric acid works as performing the role of perfect catalyst. Citric acid is widely used because it is nontoxic, cheap, safe to handle, and easily biodegradable. There are reports in the literature on the use of Citric acid as catalyst in organic synthesis ${ }^{29}$.

In continuation of our ongoing programme to develop new synthetic green protocols and our interest in the field of microwave assisted organic reactions ${ }^{30-31}$, in this report we represent a highly efficient route for the regeneration of carbonyl compounds from phenylhydrazones, semicarbazones and oximes from good to excellent yields catalyzed by commercially available inexpensive, mild citric acid as organocatalyst under microwave irradiation in water. (Scheme 1)

\section{MATERIALS AND METHODS}

Chemicals were purchased from Fluka, Merck and Aldrich chemicals companies. The purity determination of the substrates and products and reaction monitoring were accompanied by TLC on silica gel Polygram SILG/UV 254 plates. Melting points were determined on Perfit melting point apparatus and are uncorrected. ${ }^{1} \mathrm{HNMR}$ (Avance 300 $\mathrm{MHz}$ ) spectra were recorded in DMSO using TMS as internal standard. IR was recorded on a Thermo Nicolet Nexus 670 FT-IR Spectrometer and Finnegan MAT 1020. Panasonic co. microwave $(2450 \mathrm{MHz}$ frequency at a power output of $1000 \mathrm{~W}$ ) was used for the microwave irradiation.

General procedure for the deprotection of Carbonylcompounds from phenylhydrazones, semicarbazones, and oximes

A mixture of $0.2 \mathrm{mmol}$ phenylhydrazone, semicarbazones or oximes and citric acid $(0.2 \mathrm{mmol})$ were mixed together in water $(10 \mathrm{~mL})$ was introduced in a $50 \mathrm{~mL}$ Erlenmeyer flask and was placed in a commercial microwave oven operating at a power output of $300 \mathrm{~W}$ and irradiated for the appropriate time in 30 seconds intervals each. (Table 3). The reaction was monitored by TLC. The product extracted with Chloroform, dried over anhydrous $\mathrm{Na}_{2} \mathrm{SO}_{4}$, filter and was chromatographed

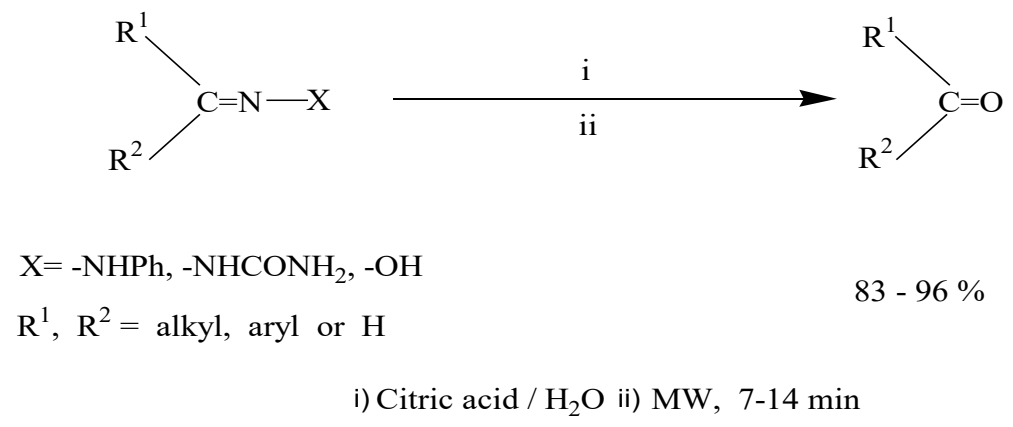

Scheme 1: Regeneration of carbonyl compounds from Phenylhydrazones, semicarbazones, and oximes in the presence of Citric acid. 
on silica gel (eluted by ethyl acetate: hexane 2:8) to afford the corresponding products in good to excellent yields (Table 3). All the products were characterized by their physical constants, IR, NMR spectra and comparison with authentic samples. The phenylhydrazones, semicarbazones and oximes were prepared by standard procedures ${ }^{32}$.

\section{Spectral data of some selected compounds} Benzaldehyde (Table 3, Entry 1)

IR (KBr): 3368,1710 cm-1; ${ }^{1} \mathrm{HNMR}$ (300
$\left.\mathrm{MHz}, \mathrm{CDCl}_{3}\right) \delta: 6.60-6.70(\mathrm{~m}, 1 \mathrm{H}, \mathrm{H}$ arom),6.92-7.15 (m, 2H, Harom), 7.68-7.72 (d, 2H, Harom), 9-10 (s, $1 \mathrm{H})$; Mass: $\mathrm{m} / \mathrm{z}\left(\mathrm{M}^{+}\right): 106$.

\section{Acetophenone (Table 3, Entry 7)}

IR (KBr): 3354, 2894, $1690 \mathrm{~cm}^{-1} ;{ }^{1}$ HNMR $\left(300 \mathrm{MHz}, \mathrm{CDCl}_{3}\right) \delta: 2.35\left(\mathrm{~s}, 3 \mathrm{H}, \mathrm{COCH}_{3}\right), 6.62-6.75$ (m, 1H, Harom), 6.92-7.10 (m, 1H, Harom), 7.757.92 (d, 2H, H arom);Mass; m/z (M+):120

Table 1: The effect of different molar quantities of catalysts on time and yields for the regeneration of benzaldehyde from benzaldehyde phenylhydrazone under MW irradiation in water

\begin{tabular}{lcccccccccccc}
\hline Entry & Catalyst(mmol)* $^{*}$ & \multicolumn{1}{c}{ Time (min) } & \multicolumn{8}{c}{ Yield (\%) } \\
& & A & B & C & D & E & A & B & C & D & E & \\
\hline 1 & 0.01 & 14 & 19 & 19 & 20 & 19 & 68 & 40 & 45 & 40 & 41 & \\
2 & 0.03 & 13 & 18 & 19 & 19 & 20 & 67 & 55 & 58 & 54 & 48 & \\
3 & 0.05 & 13 & 18 & 17 & 17 & 19 & 76 & 58 & 60 & 60 & 58 & \\
4 & 0.1 & 10 & 16 & 16 & 17 & 19 & 95 & 61 & 65 & 62 & 60 & \\
5 & 0.2 & 7 & 15 & 17 & 19 & 19 & 96 & 67 & 68 & 66 & 64 & \\
6 & 0.5 & 7 & 15 & 17 & 18 & 19 & 96 & 68 & 69 & 67 & 67 & \\
\hline
\end{tabular}

$\mathrm{A}=$ Citric acid, $\mathrm{B}=$ Glycine, $\mathrm{C}=$ Succinic acid, $\mathrm{D}=\mathrm{Oxalic}$ acid, $\mathrm{E}=$ Urea

Table 2: Regeneration of phenylhydrazones, semicarbazones and oximes by citric acid $(A)$ in water, comparison with different catalysts

( $0.2 \mathrm{mmol}$ ) under microwave irradiation and room temperature

\begin{tabular}{lcccc}
\hline Entry & Substrate* $^{*}$ & Catalyst $^{* *}$ & MW ( min / \%) & R.T(h/\%) \\
\hline 1 & $\mathrm{~S}_{1}$ & $\mathrm{~A}$ & $7.0 / 96$ & $2.0 / 60$ \\
2 & $\mathrm{~S}_{2}$ & $\mathrm{~A}$ & $7.0 / 95$ & $2.5 / 67$ \\
3 & $\mathrm{~S}_{3}$ & $\mathrm{~A}$ & $7.0 / 95$ & $2.5 / 64$ \\
4 & $\mathrm{~S}_{1}$ & $\mathrm{~B}$ & $15 / 68$ & $2.5 / 58$ \\
5 & $\mathrm{~S}_{2}$ & $\mathrm{~B}$ & $14 / 72$ & $3.0 / 59$ \\
6 & $\mathrm{~S}_{3}$ & $\mathrm{~B}$ & $13 / 65$ & $3.0 / 55$ \\
7 & $\mathrm{~S}_{1}$ & $\mathrm{C}$ & $17 / 67$ & $2.5 / 60$ \\
8 & $\mathrm{~S}_{2}$ & $\mathrm{C}$ & $17 / 70$ & $2.5 / 48$ \\
9 & $\mathrm{~S}_{3}$ & $\mathrm{C}$ & $16 / 65$ & $2.5 / 43$ \\
10 & $\mathrm{~S}_{1}$ & $\mathrm{D}$ & $18 / 67$ & $2.0 / 55$ \\
11 & $\mathrm{~S}_{2}$ & $\mathrm{D}$ & $18 / 70$ & $2.0 / 48$ \\
12 & $\mathrm{~S}_{3}$ & $\mathrm{D}$ & $16 / 69$ & $2.5 / 55$ \\
13 & $\mathrm{~S}_{1}$ & $\mathrm{E}$ & $16 / 67$ & $3.5 / 54$ \\
14 & $\mathrm{~S}_{2}$ & $\mathrm{E}$ & $18 / 70$ & $3.5 / 48$ \\
15 & $\mathrm{~S}_{3}$ & $\mathrm{E}$ & $19 / 69$ & $3.5 / 52$ \\
\hline
\end{tabular}

${ }^{*} \mathrm{~S}_{1}: \mathrm{C}_{6} \mathrm{H}_{5}=\mathrm{NNHPh}, \mathrm{S}_{2}: \mathrm{C}_{6} \mathrm{H}_{5} \mathrm{C}=\mathrm{NNHCONH}_{2}, \mathrm{~S}_{3}: \mathrm{C}_{6} \mathrm{H}_{5} \mathrm{C}=\mathrm{NOH}$

"* $\mathrm{B}=$ Glycine, $\mathrm{C}=$ Succinic acid, $\mathrm{D}=$ Oxalic acid, $\mathrm{E}=$ Urea 
Cyclohexanone (Table 3, Entry 9)

IR (KBr): 1690, $2780 \mathrm{~cm}^{-1} ;{ }^{1} \mathrm{HNMR}(300$ $\left.\mathrm{MHz}, \mathrm{CDCl}_{3}\right) \delta: 2.45-2.68(\mathrm{~m}, 4 \mathrm{H}), 2.82-2.94(\mathrm{~m}, 2 \mathrm{H})$, 4.34- $4.62(\mathrm{~m}, 4 \mathrm{H})$; Mass: $\mathrm{m} / \mathrm{z}\left(\mathrm{M}^{+}\right): 98$

Methyl Ethyl Ketone ( Table 3, Entry 11)

IR (KBr): 1697, $2798 \mathrm{~cm}^{-1}$; ${ }^{1} \mathrm{HNMR}$ : $(300$ $\left.\mathrm{MHz}, \mathrm{CDCl}_{3}\right) \delta: 2.01(\mathrm{~s}, 3 \mathrm{H}), 2.1-2.4(\mathrm{q}, 2 \mathrm{H}), 0.9-1.00$ (t, 3H); Mass: $\mathrm{m} / \mathrm{z}\left(\mathrm{M}^{+}\right): 72$
Benzophenone (Table 3, Entry 23)

IR (KBr): $1696 \mathrm{~cm}^{-1} ;{ }^{1} \mathrm{HNMR}(300 \mathrm{MHz}$, $\left.\mathrm{CDCl}_{3}\right) \quad \delta: 7.5-8.0\left(\mathrm{~m}, 10 \mathrm{H}\right.$, arom); Mass: $\mathrm{m} / \mathrm{z}\left(\mathrm{M}^{+}\right)$: 182.

\section{RESULTS AND DISCUSSION}

In the present study we broaden the scope of the citric acid as catalyst for the regeneration

Table 3: Regeneration of carbonyl compounds from phenylhydrazones (entries1-10), semicarbazones (entries11-20), and oximes (entries 21-30) using citric acid in water (10 mL) under microwave irradiation

\begin{tabular}{|c|c|c|c|c|c|}
\hline Entry & Substrate & $\begin{array}{l}\text { MW/Time } \\
\text { (min) }\end{array}$ & Product $^{a}$ & $\begin{array}{l}\text { b.p/m. } \\
p^{c}\end{array}$ & $\begin{array}{l}\text { Yield } \\
(\%)^{b}\end{array}$ \\
\hline 1 & $\mathrm{C}_{6} \mathrm{H}_{5} \mathrm{CH}=\mathrm{NNHPh}$ & 7 & $\mathrm{C}_{6} \mathrm{H}_{5} \mathrm{CH}=\mathrm{O}$ & 179 & 96 \\
\hline 2 & $2-\mathrm{NO}_{2} \mathrm{C}_{6} \mathrm{H}_{4} \mathrm{CH}=\mathrm{NNHPh}$ & 8 & 2- $\mathrm{NO}_{2} \mathrm{C}_{6} \mathrm{H}_{4} \mathrm{CH}=\mathrm{O}$ & 44 & 89 \\
\hline 3 & $4-\mathrm{NO}_{2} \mathrm{C}_{6} \mathrm{H}_{4}^{4} \mathrm{CH}=\mathrm{NNHPh}$ & 8 & 4- $\mathrm{NO}_{2} \mathrm{C}_{6} \mathrm{H}_{4} \mathrm{CH}=\mathrm{O}$ & 106 & 90 \\
\hline 4 & $2,6-\mathrm{Cl}_{2} \mathrm{C}_{6} \mathrm{H}_{4} \mathrm{CH}=\mathrm{NNHPh}$ & 9 & $2,6-\mathrm{Cl}_{2}^{2} \mathrm{C}_{6} \mathrm{H}_{4}^{4} \mathrm{CH}=\mathrm{O}$ & 71 & 87 \\
\hline 5 & $2-\mathrm{HOC}_{6} \mathrm{H}_{4} \mathrm{CH}=\mathrm{NNHPh}$ & 8 & $2-\mathrm{HOC}_{6} \mathrm{H}_{4} \mathrm{CH}=\mathrm{O}$ & 197 & 78 \\
\hline 6 & $\mathrm{C}_{2} \mathrm{H}_{5} \mathrm{C}\left(\mathrm{CH}_{3}\right)=\mathrm{NNHPh}$ & 13 & $\mathrm{C}_{2} \mathrm{H}_{5} \mathrm{C}\left(\mathrm{CH}_{3}\right)=\mathrm{O}$ & 80 & 84 \\
\hline 7 & $\mathrm{C}_{6} \mathrm{H}_{5} \mathrm{C}\left(\mathrm{CH}_{3}\right)=\mathrm{NNHPh}$ & 9 & $\mathrm{C}_{6} \mathrm{H}_{5} \mathrm{C}\left(\mathrm{CH}_{3}\right)=\mathrm{O}$ & 202 & 92 \\
\hline 8 & $\left(\mathrm{C}_{6} \mathrm{H}_{5}\right)_{2} \mathrm{C}=\mathrm{NNHPh}$ & 9 & $\left(\mathrm{C}_{6} \mathrm{H}_{5}\right)_{2} \mathrm{C}=\mathrm{O}$ & 49 & 90 \\
\hline 9 & $\mathrm{C}_{6} \mathrm{H}_{11} \mathrm{C}=\mathrm{NNHPh}$ & 14 & $\mathrm{C}_{6} \mathrm{H}_{11} \mathrm{C}=\mathrm{O}$ & 156 & 83 \\
\hline 10 & 4- $\mathrm{BrC}_{6} \mathrm{H}_{4} \mathrm{CH}=\mathrm{NNHPh}$ & 9 & $4-\mathrm{BrC}_{6} \mathrm{H}_{4} \mathrm{CH}=\mathrm{O}$ & 67 & 85 \\
\hline 11 & $\mathrm{C}_{2} \mathrm{H}_{5} \mathrm{C}\left(\mathrm{CH}_{3}\right)=\mathrm{NNHCONH}_{2}$ & 14 & $\mathrm{C}_{2} \mathrm{H}_{5} \mathrm{C}\left(\mathrm{CH}_{3}\right)=\mathrm{O}$ & 80 & 88 \\
\hline 12 & $\mathrm{C}_{6}^{2} \mathrm{H}_{5} \mathrm{C}\left(\mathrm{CH}_{3}\right)=\mathrm{NNHCONH}_{2}^{2}$ & 10 & $\mathrm{C}_{6}^{2} \mathrm{H}_{5}^{5} \mathrm{C}\left(\mathrm{CH}_{3}\right)=\mathrm{O}$ & 202 & 92 \\
\hline 13 & $\left(\mathrm{C}_{6} \mathrm{H}_{5}\right)_{2} \mathrm{C}=\mathrm{NNHCONH}_{2}$ & 9 & $\left(\mathrm{C}_{6} \mathrm{H}_{5}\right)_{2} \mathrm{C}=\mathrm{O}$ & 49 & 90 \\
\hline 14 & $\mathrm{C}_{6} \mathrm{H}_{11} \mathrm{C}=\mathrm{NNHCONH}_{2}{ }^{2}$ & 14 & $\mathrm{C}_{6} \mathrm{H}_{11} \mathrm{C}=\mathrm{O}$ & 156 & 83 \\
\hline 15 & $4-\mathrm{BrC}_{6} \mathrm{H}_{4} \mathrm{CH}=\mathrm{NNHCONH}_{2}$ & 12 & $4-\mathrm{BrC}_{6} \mathrm{H}_{4} \mathrm{CH}=\mathrm{O}$ & 67 & 87 \\
\hline 16 & $\mathrm{C}_{6} \mathrm{H}_{5} \mathrm{CH}=\mathrm{NNHCONH}_{2}$ & 7 & $\mathrm{C}_{6} \mathrm{H}_{5} \mathrm{CH}=\mathrm{O}$ & 179 & 95 \\
\hline 17 & $2-\mathrm{NO}_{2} \mathrm{C}_{6} \mathrm{H}_{4} \mathrm{CH}=\mathrm{NNHCONH}$ & 8 & 2- $\mathrm{NO}_{2} \mathrm{C}_{6} \mathrm{H}_{4} \mathrm{CH}=\mathrm{O}$ & 44 & 86 \\
\hline 18 & 4- $\mathrm{NO}_{2} \mathrm{C}_{6} \mathrm{H}_{4}^{4} \mathrm{CH}=\mathrm{NNHCONH}$ & 8 & $4-\mathrm{NO}_{2} \mathrm{C}_{6} \mathrm{H}_{4}^{4} \mathrm{CH}=\mathrm{O}$ & 106 & 89 \\
\hline 19 & $2,6-\mathrm{Cl}_{2} \mathrm{C}_{6} \mathrm{H}_{4}^{4} \mathrm{CH}=\mathrm{NNHCONH}_{2}$ & 9 & $2,6-\mathrm{Cl}_{2} \mathrm{C}_{6} \mathrm{H}_{4}^{4} \mathrm{CH}=\mathrm{O}$ & 71 & 83 \\
\hline 20 & $2-\mathrm{HOC}_{6} \mathrm{H}_{4} \mathrm{CH}=\mathrm{NNHCONH}_{2}$ & 8 & $2-\mathrm{HOC}_{6} \mathrm{H}_{4} \mathrm{CH}=\mathrm{O}$ & 197 & 73 \\
\hline 21 & $\mathrm{C}_{2} \mathrm{H}_{5} \mathrm{C}\left(\mathrm{CH}_{3}\right)=\mathrm{NOH}$ & 13 & $\mathrm{C}_{2} \mathrm{H}_{5} \mathrm{C}\left(\mathrm{CH}_{3}^{4}\right)=\mathrm{O}$ & 80 & 88 \\
\hline 22 & $\mathrm{C}_{6} \mathrm{H}_{5} \mathrm{C}\left(\mathrm{CH}_{3}\right)=\mathrm{NOH}$ & 9 & $\mathrm{C}_{6} \mathrm{H}_{5} \mathrm{C}\left(\mathrm{CH}_{3}\right)=\mathrm{O}$ & 202 & 92 \\
\hline 23 & $\left(\mathrm{C}_{6} \mathrm{H}_{5}\right)_{2} \mathrm{C}=\mathrm{NOH}$ & 10 & $\left(\mathrm{C}_{6} \mathrm{H}_{5}\right)_{2} \mathrm{C}=\mathrm{O}$ & 49 & 90 \\
\hline 24 & $\mathrm{C}_{6} \mathrm{H}_{11} \mathrm{C}=\mathrm{NOH}$ & 14 & $\mathrm{C}_{6} \mathrm{H}_{11}{ }^{5 / 2}=\mathrm{O}$ & 156 & 83 \\
\hline 25 & $4-\mathrm{BrC}_{6} \mathrm{H}_{4} \mathrm{CH}=\mathrm{NOH}$ & 9 & $4-\mathrm{BrC}_{6} \mathrm{H}_{4} \mathrm{CH}=\mathrm{O}$ & 67 & 87 \\
\hline 26 & $\mathrm{C}_{6} \mathrm{H}_{5} \mathrm{CH}=\mathrm{NOH}$ & 7 & $\mathrm{C}_{6} \mathrm{H}_{5} \mathrm{CH}=\mathrm{O}$ & 179 & 95 \\
\hline 27 & $2-\mathrm{NO}_{2} \mathrm{C}_{6} \mathrm{H}_{4} \mathrm{CH}=\mathrm{NOH}$ & 8 & 2- $\mathrm{NO}_{2} \mathrm{C}_{6} \mathrm{H}_{4} \mathrm{CH}=\mathrm{O}$ & 44 & 86 \\
\hline 28 & $4-\mathrm{NO}_{2} \mathrm{C}_{6}{ }_{6} \mathrm{H}_{4}^{4} \mathrm{CH}=\mathrm{NOH}$ & 8 & $4-\mathrm{NO}_{2}^{2} \mathrm{C}_{6}^{6} \mathrm{H}_{4}^{4} \mathrm{CH}=\mathrm{O}$ & 106 & 89 \\
\hline 29 & $2,6-\mathrm{Cl}_{2} \mathrm{C}_{6} \mathrm{H}_{4} \mathrm{CH}=\mathrm{NOH}$ & 10 & $2,6-\mathrm{Cl}_{2} \mathrm{C}_{6} \mathrm{H}_{4} \mathrm{CH}=\mathrm{O}$ & 71 & 83 \\
\hline 30 & $2-\mathrm{HOC}_{6} \mathrm{H}_{4} \mathrm{CH}=\mathrm{NOH}$ & 8 & $2-\mathrm{HOC}_{6} \mathrm{H}_{4} \mathrm{CH}=\mathrm{O}$ & 197 & 76 \\
\hline
\end{tabular}

a; All the products are identified by comparing IR, NMR and Physical constants with those authentic samples. ;Yields refer to isolated products. ${ }^{\mathrm{c}}$; Referenc ${ }^{32}$ 
of carbonyl compounds from phenylhydrazones, semicarbazones and oximes under microwave irradiations in water. In order to check the role of catalyst we studied the efficiency of regeneration of benzaldehyde from benzaldehyde phenylhydrazone in water as a model reaction in absence of catalyst under microwave irradiation. After 20 minutes of irradiation there is no detection of carbonyl compounds was observed. Subsequently to investigate the suitable catalyst and the best molar quantities we carried out the model reaction of benzaldehyde phenylhydrazone in presence of different molar quantities of various catalysts for example citric acid, glycine, succinic acid, or urea. From the results obtained in Table 1, we found that $(0.2 \mathrm{mmol})$ citric acid is emerged as the best catalyst of choice in terms of reaction kinetics and product yield (entry 6, Table 1 ).

Then the model reaction has been carried out by stirring a mixture of benzaldehyde phenylhydrazone $(0.2 \mathrm{~m} \mathrm{~mol})$ and $0.2 \mathrm{~m} \mathrm{~mol}$ of the catalyst in $10 \mathrm{~mL}$ of water at room temperature as well as microwave irradiation. From the results shown in Table 2 we have found that using citric acid under micro wave irradiation afforded the highest yield ( entry $196 \%$, Table 2). Similarly, we run the same reaction condition on benzaldehyde semicarbazone and benzaldehyde oxime in the presence of various catalysts, such as citric acid, succinic acid, glycine, oxalic acid, or urea. The best results were obtained by using citric acid as catalyst comparable with that obtained in the presence of other previously mentioned catalysts (Table 2 ). Then the model reaction has been carried out by stirring a mixture of benzaldehyde phenylhydrazone $(0.2 \mathrm{mmol})$ and $0.2 \mathrm{mmol}$ of the catalyst in $10 \mathrm{~mL}$ of water at room temperature as well as microwave irradiation. From the results shown in Table 2 we have found that using citric acid under micro wave irradiation afforded the highest yield ( entry $196 \%$, Table 2). Similarly, we run the same reaction condition on benzaldehyde semicarbazone and benzaldehyde oxime in the presence of various catalysts, such as citric acid, succinic acid, glycine, oxalic acid, or urea.The best results were obtained by using citric acid as catalyst comparable with that obtained in the presence of other previously mentioned catalysts( Table 2 ).

Finally a series of experiments were performed by taking different derivatives of phenylhydrazones, semicarbazones and oximes for the regeneration of carbonyl compounds under microwave irradiations (Table 3). Regeneration of aliphatic phenylhydrazones, semicarbazones, and oximes normally requires longer reaction times (entries 6, 9, 11, 14, 21, 24). Therefore this methodology offers a simple, inexpensive and selective route for converting phenylhydrazones, semicarbazones and oximes to the corresponding carbonyl compounds.

\section{CONCLUSION}

In conclusion we have developed a clean and environmentally friendly alternative protocol for the regeneration of carbonyl compounds from phenylhydrazones, semicarbazones and oximes in good to excellent yields under microwave irradiations and water as a green solvent by using commercially available inexpensive biodegradable citric acid. The present protocol has several advantages; mild reaction conditions, reduced reaction times operational and experimental simplicity. We believe that the new methodology will be a valuable addition for the regeneration of carbonyl compounds from phenylhydrazones, semicarbazones and oximes.

\section{REFERENCES}

1. Whitesell, J. K.; Whitesell, M. A. Synthesis 1983, 7, 517.

2. Green, T.W.; Wuts, P. G. M. Protective groups in organic synthesis, (John Wiley, New York) 1991, 175.

3. Shriner, R.L.; Fusion R.C.; Curtin, D.Y.; Morrill T.C. The Systematic Identification of Organic compounds, $6^{\text {th }}$ edn, (JohnWiley, New York), 1980.

4. Varma, R.S.; Meshram, H.M. Tetrahedron
Lett. 1997, 38, 973.

5. Beldar, A.G.; Sharma, M. E. J. Chem. 2011, 8, 288.

6. Varma, R.S.; Dahiya, R.; Saini, R. K. Tetrahedron Lett. 1997, 38, 8819.

7. Bose, S.D.; Srinivas, P.; Synlett. 1998, 9, 977.

8. Mathur, L.; Ranga, V.; Goswami, G.; Agarwal, S.; Sharma, P. K.; Eur.Chem. Bull., 2014, 3, 823. 
9. Demir, A.S. Tanyeli,C.; Altinel, E.; Tetrahedron Lett. 1997, 38, 7267.

10. Perumal, P.T.; Anniyappan, M.; Muralidharan, D.; J. Chem. Sci. 2004, 116, 261.

11. Fan, X.; Zhanga, X.; Zhanga,Y. J. Chem. Res (S), 2004, 290.

12. Bhatnagar, I.; George, M.V. J. Org. Chem, 1967, 32, 2252.

13. Laszlo, P.; Polla, S.; Synthesis, 1985, 4, 439.

14. Hosseinzadeh, R.; Tajbakhsh, M.; Niaki, M.Y. Tetrahedron Lett. 2002, 43, 9413.

15. Ram, R.N.; Varsh, K.; Tetrahedron Lett. 1991, 32, 5829 .

16. Hajipour, A.R.; Adibi, H.; Ruoho, A.E. J.Org. Chem. 2003, 68, 4553.

17. Srinivas, K.V.N.S.; Das, B.; J. Chem. Res. (S), 2002, 110, 556.

18. Chakrabarty, M.; Khasnobis, S. Synth. Commun. 1998, 28, 1361.

19. Hakimi, M.; Feizi, N.; Hassani, H.; Vahedi, H.; Thomas, P. S. Synth.Commun. 2010 1, 725.

20. De, S.K.; Tetrahedron Lett. 2003, 44, 9055.

21. Khazaei, A.; Vaghei, R.G. Molecules, 2002, 7, 465.

22. (a) Mc killop, A.; Hunt, J. D.; Taylor, E. C.; Kienzle F. Tetrahedron Lett. 1970, 11, 5275

(b) Mc killop, A.; Hunt, J. D.; Naylor, R. D.; Taylor, E. C. J. Am.Chem. Soc. 1971, 93, 4918.

23. Yukawa, Y.; Sakai, M.; Suzuki, S. Bull. Chem. Soc. Jpn. 1966, 39, 2266.

24. Bendale, P. M.; Khdilkar, B.M. Tetrahedron Lett. 1998, 39, 5867.

25. Lalitha, A. Sivakumar, K.; Parameswaran, K.; Pitchumani, K. K.; Srinivasan, C. Res. Lett. Org. Chem. 2009, 4, 1.

26. (a) Varma, R.S. Green Chem., 1999, 43. (b) Loupy, A. Microwaves in organic synthesis, (Wiley-VCH,Weinheim), 2002.(c) Hayes B.L. Microwave synthesis (C E M Publishing, Mathews) 2002 (d) Banik, B. K. Heterocycl. lett. 2014, 4, 463. (e) Wanga, P.; Maa,C.; Chenb, S.; Zhub, S.; Loua, Z.; Wanga, H. J. Clean. Prod. 2014, 79, 265.(f) E.M.H. Abbasa, E.M.H.; Gomhab, S. M.; Farghaly,T.A. Arabian J. of. Chem. 2014, 7, 623.

27. (a)Yadav,L.D.S.; Singh, A.; Tetrahedron Lett. 2003, 44, 5637. (b) Mitra, A. K. Banerjee, K. Synlett 2003, 10, 1509. (c) Pansare, D. N.; Shinde, D. B. Tetrahedron Lett. 2014, 5, 1107. (d) Zakeria, M.; Nasefa, M. M.; Lotf, E. A. J. Mol. Liq. 2014, 199, 267 (e) Khajuria, R. Saini, Y.; Kapoor, K.K. Tetrahedron Lett. 2013, 42, 5699.

28. (a) Mitra, A.K.; De, A.; Karchaudhuri, N. Synlett, 1998, 12, 1345. (b) Mitra, A.K.; De, A.; Karchaudhuri, N.; J. Chem. Res (S), 1999, 320, 56 (c) Mitra, A.K.; De, A.; Karchaudhuri, N.; Synth Commun, 1999, 29, 2731.

29. (a) Seyedi, N.; Kalantari, M. J. Sci. Islam. Repub. Iran 2013,24, 205.(b) Ramu, E.; Kotra, V.; Bansal, N.; Varala, R.; Adapa, S.R. Rasayan. J.Chem.2008, 1, 188. (c) Ding, Q.S.; Zhang, J.L.; Chen, J.X.; Liu, M.C.; Ding, J.C .; Wu, H.Y. Tandem J. Heterocyclic Chem. 2012, 49, 375 (d) Sajjadifar, S.; Zolfigol, M.A.; Chehardoli,G.; Miri,S.; P.Moosav, P. I. J. Chem. Tech. Res. 2013, 5, 422 (e) Ahmed, M.Z.; Patel, N.T.; Shaikh, K. A .; Baseer, M. A.; Shaikh, S.; Patti, V. A Elixier. Org. Chem. 2010, 43, 6583.

30. (a) Abd El-Rahman, N.M.; El-kateb, A.A.; Mady, M.F.; Synthetic commun. 2007, 37, 3961(b)Abd El-Rahman,N.M.; Fawzy,N.M.; Zaki,E.A. International Journal of Pharm. Tech. Research, 2009, 1, 857 (c) Saleh, T.S.; Elkholy, Y.M.; Mohamed, A.A.; Abd El-Rahman, N.M. Egypt. J. Chem. 2009 52, 277(d) Saleh, T.S.; Abd El-Rahman, N.M.; Assaker, R.S.A. Green chem. Let. rev. 2012, 5, 315. (e) El-Kateb, A.A.; Abd El-Rahman, N.M.; Saleh, T.S. El-Kateb, A.A.; Abd ElRahman, N.M.; T.S. Saleh, Ali, H.; El- Dosoky, A.Y.; Awad, G.E.A. J. of Appl. Sci. Res. 2012, 7, 3393 (f) Abd El-Rahman, N.M.; Borik, R.M. World Applied Sciences Journal, 2014, 31, 1.

31. (a) Nasreen, A. Tetrahedron Lett. 2013, 54, 3797 (b) Nasreen, A. Asian journal of Chemistry, 2013, 25, 7535. (c) Varala, R.; Nasreen, A.; Ramu, E.; Adapa, S.R. Tetrahedron Lett. 2007, 48, 69 (d) Varala, R.; Nasreen, A.; Adapa, S.R. Can. J. Chem., 2007, 85, 148. (e) Nasreen, A.; Borik, R.M. Oriental Journal of Chemistry 2014, 30, 761. (f) Varala, R.; Nasreen, A.;Adapa S.R. J. Heterocycl. Chem. 2007, 44, 1. (g) Nasreen, A.; Adapa, S.R. Organic Preparations and Procedures International, 2000, 32, 373.

32. Vogel, A. I. Textbook of Practical Organic Chemistry; $5^{\text {th }}$ ed.; ELBS and Longman: London, 1989, 1258-1259. 